\title{
Methodology for compiling a microbial resistance passport for dairy farms
}

\author{
A. S. Krivonogova ${ }^{1}$, I. M. Donnik ${ }^{2}$, A. G. Isaeva ${ }^{2 \varpi}$, K. V. Moiseeva ${ }^{2}$ \\ ${ }^{1}$ Ural Federal Agrarian Research Center of the Ural Branch of the Russian Academy of Sciences, Ekaterinburg, \\ Russia \\ ${ }^{2}$ Ural State Agrarian University, Ekaterinburg, Russia \\ 凶E-mail: isaeva.05@bk.ru
}

Abstract. The article presents the results of the work, the purpose of which was to create a microbial resistance passport for a dairy farm. Methods. Conducted studies of opportunistic pathogenic microflora in various technological areas, there were taken samples of feed, water, air, bedding, washings from the wool and mucous membranes of cows and calves, washings from surfaces, fences, technological equipment, and livestock care equipment. There were determined the frequency of occurrence and the proportion of microorganisms in the structure of the opportunistic microbiocenosis. Results. It was found that on all surveyed dairy farms, the nucleus of opportunistic microbiocenosis was represented by Ent. faecium, Ent. faecalis, S. aureus, S. epidermidis, S. saprophyticus, P. aeruginosa, P. vulgaris, P. mirabilis, Echerichia coli, Aspergillus spp. and C. albicans the frequency of occurrence of these microorganisms in samples exceeded $15 \%$. We studied the two-year dynamics of the microbiocenoses composition on farms. It was found that the main nucleus of the microbiocenosis remained stable in species composition, and the percentage of stocks was subject to slight changes. There were carried out he studies of the sensitivity of all detected stocks to antibiotics, based on the results of which were compiled resistances profiles, methods were developed for the analysis of general indicators of microbial resistance throughout the enterprise and in individual technological areas, and were established the ways of spreading resistant stocks on the farm. On the basis of four criteria, including the effectiveness of the antibiotic against the target microorganism, the effectiveness against the non-target microorganism in vitro, the degree of growth inhibition, the minimum inhibitory concentration, the antibiotic susceptibility analysis of the main pathogenic microorganisms was carried out in points. The results obtained were used to predict the expected effectiveness of antibiotics in different technological areas of the farm. The novelty of the researches is in the development of an algorithm for compiling a passport of microbial resistance of a dairy farm, which includes analysis of opportunistic microbiocenosis, analysis of antibiotic sensitivity of microorganisms and prediction of the expected effectiveness of antibacterial drugs.

Keywords: microbial resistance, opportunistic microflora, antibiotic sensitivity, antibiotic resistance, dairy farm, cattle, microbial resistance passport, monitoring of microbial resistance.

For citation: Krivonogova A. S., Donnik I. M., Isaeva A. G., Moiseeva K. V. Methodology for compiling a microbial resistance passport for dairy farms // Agrarian Bulletin of the Urals. 2020. No. 09 (200). Pp. 42-47. DOI: ...

Paper submitted: 20.07.2020.

\section{Introduction}

According to the World Health Organization, antimicrobial resistance of microorganisms is currently among the urgent threats to the well-being of humanity and is a multifaceted problem affecting the entire society. To combat the development and spread of antimicrobial resistance (AMR), there are required concerted actions at all executive levels - from first-line specialists to the leadership of countries and international associations [1]. A long period of uncontrolled use of antimicrobial drugs in healthcare, veterinary medicine and agriculture has led to the spread of forms of microorganisms, including pathogens of infectious diseases with genetic characteristics that determine resistance to antimicrobial drugs, including antibiotics, anti-tuberculosis, antiviral, antiparasitic and antifungal drugs [2]. In May 2015, the World Health Assembly approved a global action plan to tackle antimicrobial resistance, including antibiotic resistance, which is the most pressing trend in drug resistance. Monitoring of microflora resistance and antibiotic control in veterinary medicine and agriculture are among the main areas of work to prevent AMR [3]. In 2017, the Russian Federation adopted the Strategy for preventing the spread of antimicrobial resistance. Among the main reasons for the emergence and spread of antimicrobial resistance related to veterinary medicine and agriculture, are the irrational and (or) uncontrolled use of antimicrobial drugs, insufficient diagnosis of resistance of microorganisms to drugs, a violation of the composition of the normal microbiota of animals; lack of AMR monitoring mechanisms. [2]. The World Veterinary Association points to the critical role of the line animal veterinarian. It appears to be more significant than government measures such as licensing and control. Because the use of an antibiotic in each specific case is the responsibility of the veterinarian, who determines the indications, type, dosage and regimen of the drug. At the same time, the WVA 
supports the idea that each country should have an appropriate regulatory system for licensing the control of veterinary antimicrobial drugs [4]. Responsible and reasonable use of antibiotics in veterinary medicine, declared in the Terrestrial Animal Health Code, implies an analysis of the risks of developing antimicrobial resistance in animals, control and restriction of the use of feed antibiotics, prohibition of their prophylactic intake and prescriptions without prior determination of the sensitivity of target microorganisms [5]. When tracking and registering the use of veterinary antimicrobial drugs, it is recommended to indicate the type of animals, the type of drug, periods and treatment regimens, data on the susceptibility of microorganisms, the response of animals to therapy, etc. The importance of identifying, describing, monitoring and controlling all episodes of resistance in productive animals is emphasized. [5].

In Russia, the AMR containment strategy involves, inter alia, work on diagnostics and restoration of disturbed natural microbiocenoses of humans, farm animals and plants; rational use and production control over the use of antimicrobial drugs in health and veterinary medicine; increasing the detectability of the resistance of pathogens of infectious diseases in humans, animals and plants. Monitoring the spread of antimicrobial resistance based on laboratory diagnostic data is one of the main directions of monitoring biological and chemical threats in the Russian Federation [2]. Unfortunately, today the domestic livestock sector is in economic conditions that do not allow one to immediately abandon the use of antibiotic-containing feed additives, or to conduct individual laboratory diagnostics of each animal to select the optimal antibiotic therapy. Due to the low availability of laboratory diagnostic services and a limited range of antibiotics approved in veterinary medicine, most livestock enterprises have to use the same therapy regimens for years without changing drugs and preliminary sensitivity control.

In addition, there are objective technological, environmental and biological factors that contribute to the spread of AMR in livestock populations - the placement of large livestock in a limited area, reduced mobility of animals, crowding, circulation of infectious agents, requiring treatment with antimicrobial drugs (AMP), constant stress, unfavorable ecological environment, low quality feed, etc. [6, P. 10], [7, P. 605], [8], $[9$, P. 203]. The resulting change in the balance of microorganisms in the opportunistic microbiocenosis can simultaneously be both a cause and a consequence of the spread of AMR. Replacement of non-pathogenic native microflora with pathogenic strains, increased aggressiveness and resistance of opportunistic species leads to the fact that undesirable microorganisms are firmly fixed in the core of the microbiocenosis of the animal, which in turn leads to a decrease in the general somatic and immune status, an increase in morbidity, a decrease in productivity and product quality, as well as to the spread of AMR and a decrease in the therapeutic efficacy of antibacterial drugs (ABD) [6, Pp. 9, 12], [10, P. 1], [11, Pp. 49-50]. The most common representatives of opportunistic microbiocenoses at livestock enterprises are enterococci, Pseudomonas aeruginosa, staphylococci, streptococci, Klebsiella, Escherichia coli, Proteus, which cause a large number of purulent-septic processes in animals with low immune status [8], [11, P. 48], [12], [13, P. 37], [9, P. 207]. The danger of these microorgan- isms is due to their high adaptive potential, genetic flexibility, the ability to quickly develop resistance to antimicrobial drugs [14, Pp. 210-212], [15, P. 129], [16, P. 791], [17, Pp. 30-32]. The number of cases of detection of multi-resistant strains of $\mathrm{P}$. aeruginosa, $\mathrm{S}$. aureus, E. faecium is growing every year both in the field of public health and in animal husbandry and veterinary medicine around the world [18, P. 642], [10, P. 1], [19, P. 173], [14, P. 210]. Uncontrolled, unsystematic, unjustified use of antibacterial drugs for prophylaxis and therapy in animals, especially in those with immunodeficiencies of various origins, in patients, newborns, young animals, inevitably leads to a decrease in the sensitivity of the strains that make up the permanent core of microbiocenosis [13, P. 36], [20, Pp. 312323], [17, Pp. 30-34].

As part of the work to prevent the spread of microbial resistance on livestock facilities in the Ural region, screening studies of microbiocenoses of commercial dairy farms (CDF) and pig breeding enterprises are required, which will make it possible to assess the key characteristics of opportunistic microflora, its sensitivity to antibiotics of various classes, identify episodes of resistance and analyze possible routes of spread resistant strains of microorganisms inside livestock facilities. In this regard, it seems relevant to develop a method for compiling a passport of resistance for dairy farms, including a study of the structure of opportunistic microbiocenoses, an assessment of the sensitivity of strains to antibiotics, and an analysis of the data obtained.

The purpose of the research was to develop a passport for the resistance of a dairy farm. To achieve this goal, the following tasks were implemented: development of an optimal method for studying the structure of opportunistic microbiocenoses of CDF; study of sensitivity to antibiotics and selection of the most effective screening tactics; selection of the optimal method for processing the obtained data, which most fully reflects the AMR pattern at the facility with the least resource intensity.

Methods
We investigated the opportunistic microflora of commercial dairy farms in the Ural region, the composition of the microbiocenosis, the frequency of occurrence of individual strains of microorganisms, and were determined their sensitivity to antibiotics. Samples were taken at livestock technological facilities of farms - in dairy cattle departments, maternity wards, calves, manure storage facilities, milking parlors. For microbiological analysis, washings were performed from mucous membranes, wool cover, from the udder of cows and calves, washings from equipment, feeders, drinkers, fences, surfaces, cattle care equipment; samples were taken of litter, manure, drinking water, feed components and finished feed mixture, air. At each technological site, were choosen from 10 to 60 samples. There was determined the total microbial number, was isolated a clean line, were identified microorganisms, their pathogenicity and sensitivity to antibiotics were determined by the disc diffusion method and by the method of serial dilutions according to standard methods [21]. The results obtained in the course of the research were analyzed by the methods of mathematical statistics in the STATISTICA 10 program with the calculation of the mean value, standard deviation, checking the normality using the Shapiro-Wilk test, assessing sig- 
nificant differences between the groups according to various indicators by ANOVA methods, and the Mann-Whitney test. The data obtained were used to develop and compile a passport for microbial resistance. The research is executed at the expense of a grant of the RSF (project No.18-16-00040).

\section{Results}

At the first stage, were carried out studies of the structure of microbiocenoses, were determined typical representatives of opportunistic microflora, and strains of microorganisms were analyzed according to the frequency of occurrence in samples. It was found that on the examined dairy farms of the Ural region, the generic and species composition of the microflora was relatively the same, but the structure of the microbiocenosis and the frequency of occurrence of individual strains varied depending on the enterprise and the technological site. Typical representatives of pathogenic and conditionally pathogenic microflora on CDF were: Enterococcus faecium, Enterococcus faecalis, Enterococcus durans, Pseudomonas aeruginosa, Staphylococcus aureus, Staphylococcus epidermidis, Staphylococcus epidermidis, Staphylococcus miraculum epidermidis, Staphylococcus miraculum, Proteobacterus saprophilis, Enterococcus saprophilis, Enterococcus sulphate, Enterococcus saprophilis, Enterococci farmeri, Klebsiella spp., a significant amount of Candida albicans, Aspergillus spp., Mucor spp., Penicillium spp., Fusaium spp. were also found in the samples. When analyzing the frequency of occurrence of microorganisms, the number of samples positive for a specific microorganism from each object was determined. The most common microorganisms in the samples were Enterococcus faecium $(85 \%-100 \%$ of samples depending on the object), Staphylococcus aureus ( $65 \%-75 \%$ of samples), P. aeruginosa (in $50 \%-70 \%$ of samples), Ent. faecalis (more than $40 \%-65 \%$ of samples). Among the representatives of the fungal microflora, Aspergillus spp. and Candida albicans, which were plated from more than $75 \%$ and $55 \%$, respectively. Citrobacter, Klebsiella, Bacillius subtilis were found in the samples least often. Their frequency of occurrence was on average less than $4 \%$. Separate, single microorganisms found on objects - Ent. durans, S. pneumonia, K. pneumoniae, C. freundii, C. diversus, etc. were not taken into account in the general structure of microbiocenosis, since the total frequency of their occurrence was less than $0.2-0.5 \%$. The structure of the opportunistic microbiocenosis was analyzed for each technological object, for which the proportion of each microorganism from the total number isolated at this object was calculated. Separate calculations were performed for pathogenic and potentially pathogenic microorganisms (fig. 1).

There was carried out a comparative analysis of the structure of the microbiocenosis with similar data from the previous year. The dynamics of changes was expressed in a decrease or increase in the proportion of microorganisms. It was found that, in general, the core of the microbiocenosis remained stable, the proportion of Staphylococcus aureus, enterococci, Proteus changed insignificantly. The share of Pseudomonas aeruginosa and yeast was subject to large fluctuations. Also, in a number of enterprises, there was a tendency to a decrease in the share of Aspergillus spp., Mucor spp., Penicillium spp, Fusaium spp. in samples of air, water, feed, bedding, washings from drinking bowls, feeders, animal hair for two to three years. At these facilities, work was carried out to update the ventilation and water supply systems, and the feed preparation system was modernized. Presumably, these factors influenced the contamination of objects with molds.

Analysis of the microflora of the swabs showed that in the overwhelming majority of samples (more than $93 \%$ ) taken from the wool cover of cows, the same strains were found and with the same frequency as in swabs taken from fences and surfaces of livestock buildings. A similar similarity was found for samples of litter and coat of newborn calves in the maternity ward, for washings from rags for washing cows, washings from the udder and glasses of the milking machine. This fact testifies to constant contact seeding, and in the case of antibiotic-resistant pathogenic strains, it may be one of the mechanisms of the spread of resistance. Proteus strains). Thus, vancomycin-resistant Ent strains were detected on the examined CDFs. faecium and multi-resistant P. aeruginosa strains with the same antibiotic susceptibility profile in litter samples, swabs from the genital tract of cows from maternity wards, and swabs from the mucous membranes of the mouth and nose of calves. Since the seeding of a newborn calves with strains of opportunistic microflora occurred when it came into contact with the litter, surfaces in the maternity ward. When resistant

The structure of pathogenic microbiocenosis in the maternity ward
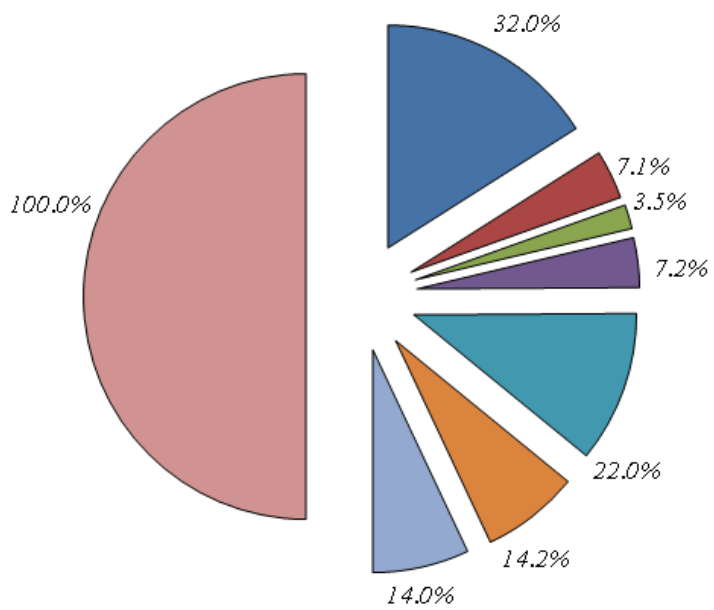

$\square$ Enterococcus faecium

$\square$ Enterococcus faecalis

$\square$ Staphylococcus aureus

$\square$ Staphylococcus saprophyticus

$\square$ Pseudomonas aeruginosa

$\square$ Proteus spp.

$\square$ Candida albicans

$\square$ sum

Fig. 1. The structure of pathogenic microbiocenosis in the maternity ward of the CDF 
strains circulate in maternity wards, the risk of contamination of calves with them increases, which, in turn, may lead to an increase in morbidity and low effectiveness of antibiotic therapy in these groups of animals. In general, P. aeruginosa, S. aureus and Ent strains prevailed in the maternity wards of the surveyed farms. faecium, in dairy cow compartments - E. coli, Ent. faecalis, S. epidermidis, S. saprophyticus and Proteus (in $86-90 \%$ of cases, P. vulgaris, and P. mirabilis averaged 10$14 \%$ of the isolated proteus strains).

At the second stage of the research, there was analyzed the sensitivity of all isolated strains to antibiotics. Antibiotics were selected from those that were most frequently used on farms over the previous three years: rifampicin (ansamycins), meropenem (carbapenems), ampicillin, amoxicillin (semisynthetic pennicillins), cefazolin (generation I cephalosporin), enrofloxacin (fluoroquinolones III) (macrolides), tetracycline (tetracyclines), vancomycin (glycopeptides). The obtained results were analyzed and was calculated the total sensitivity of the isolated strains to each antibiotic (fig. 2), as well as the total sensitivity of the main pathogenic strains to antibiotics (fig. 3).
In addition, a separate analysis was performed for all episodes of detection of resistant strains. In the microbial resistance passport of the enterprise, these data were included in the form of a table indicating the strain of the microorganism, the place of its detection (sampling), the antibiotic to which resistance was detected and antibiotics to which the maximum sensitivity was revealed for this strain.

To predict the effectiveness of antibacterial drugs at a specific technological site (maternity ward, a group of calves, heifers, dairy cattle, etc.), the sensitivity analysis of the strains found at the facility was carried out according to the system of criteria developed by us and the assessment in points. The criteria were the effectiveness of the antibiotic against the target microorganism, the effectiveness against the non-target microorganism in vitro, the degree of growth inhibition, the minimum inhibitory concentration. The results were statistically processed with the calculation of trends and analyzed the estimated efficacy of the drug against the main pathogenic microorganisms (fig. 4).

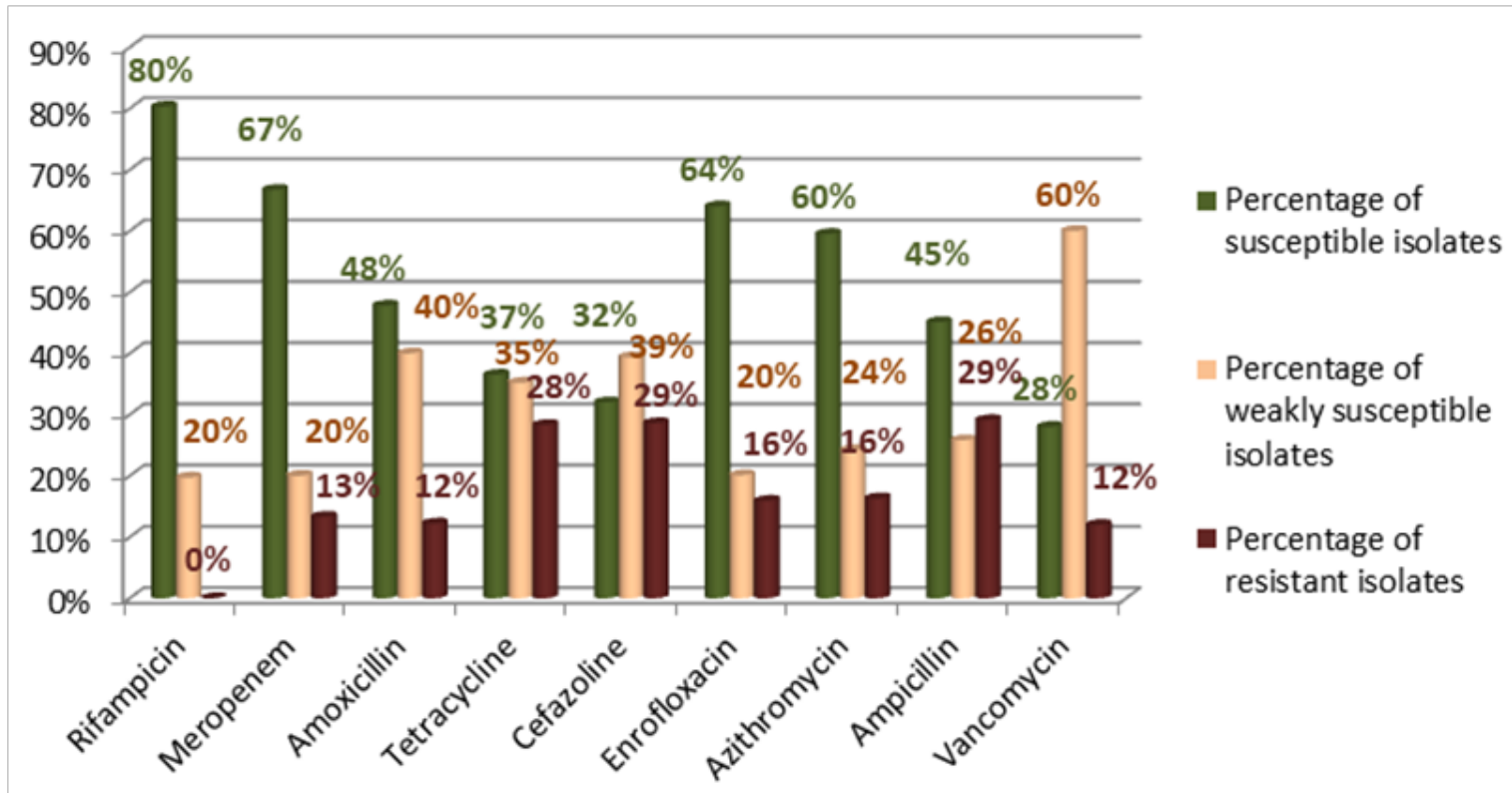

Fig. 2. Antibiotic sensitivity of all isolated strains in 1-2 months old calves section

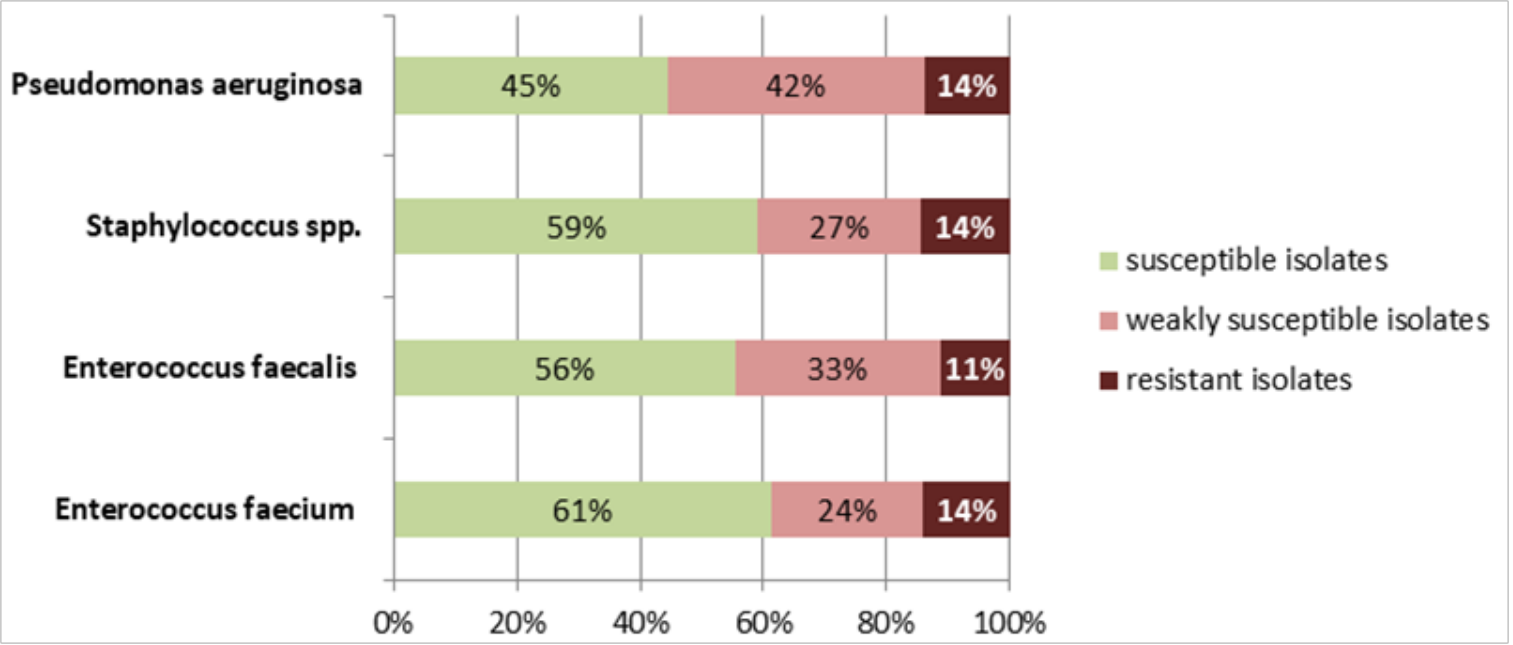

Fig. 3. Total sensitivity of microorganisms to 9 antibiotics in 1-2 months old calves section 


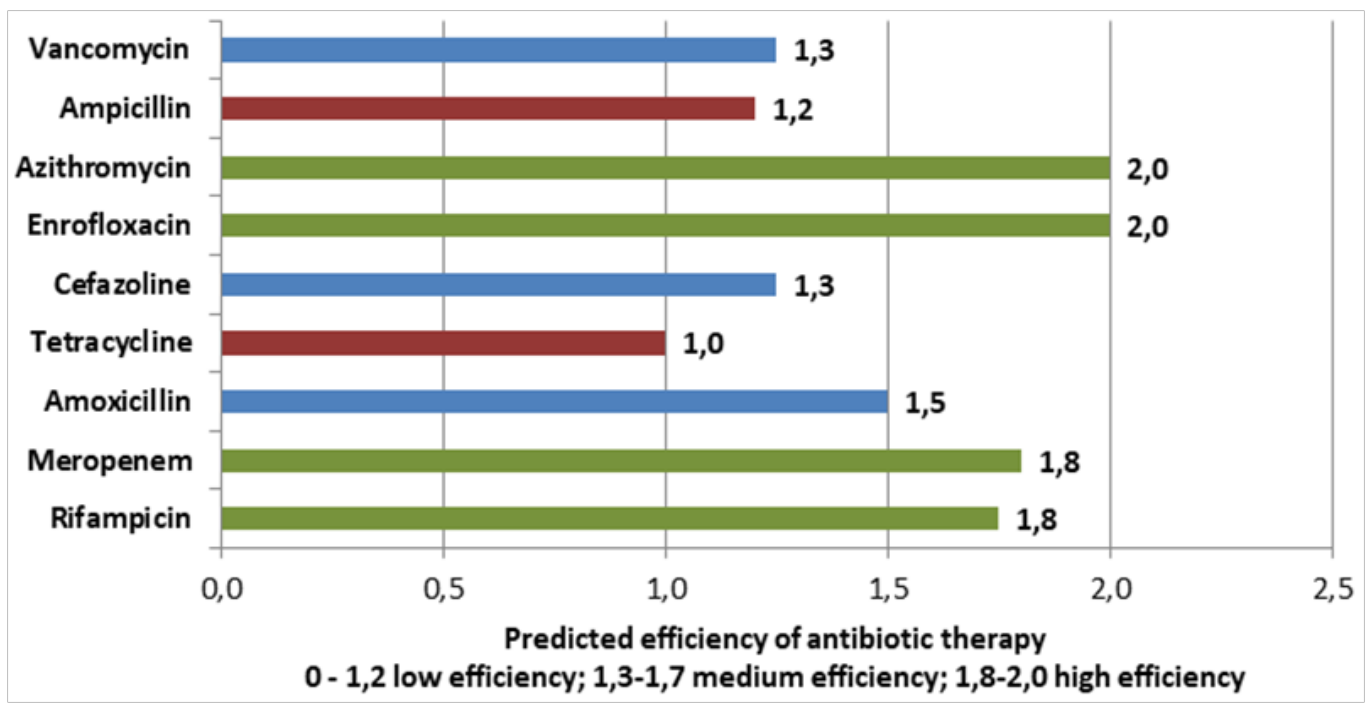

Fig. 4. Predicted antibiotic efficiency against $E$. faecium in the dairy unit on CDF

\section{Discussion and Conclusion}

Our studies have shown that the species composition of the nucleus of opportunistic microbiocenosis at various objects of dairy farms remains practically unchanged and is represented mainly by enterococci, staphylococci, Pseudomonas aeruginosa and Escherichia coli, Proteus, mold fungi, yeast. The share of a specific microorganism in the structure of the opportunistic microbiocenosis is variable and depends on the type, purpose and characteristics of the technological site. When compiling a passport of microbial resistance of MTF, it is advisable to examine each site or department, taking into account the constant contact contamination of various objects on the site. When analyzing the antibiotic sensitivity of the isolated strains in conjunction with the analysis of the structure of the opportunistic microbiocenosis and the comparison of resistance profiles, it is possible to predict the spread of resistant strains in the area and a preliminary assessment of the effectiveness of antibiotic therapy. Our proposed algorithm for compiling a passport for microbial resistance of a dairy farm involves a comprehensive study of the structure of opportunistic microbiocenosis, analysis of the antibiotic sensitivity of strains by the disc diffusion method and a dilution method with an assessment according to four criteria, identification and analysis of all episodes of resistance, calculation of the overall effectiveness of each type of antibiotic against strains found in the department or at the site, calculation of the total sensitivity of the main pathogens to the studied antibiotics, prediction of the effectiveness of antibiotics against microorganisms that make up the core of the opportunistic microbiocenosis.

Our prediction of the effectiveness of antibiotics can be used by CDF specialists to adjust treatment if it is impossible to determine the individual microbial picture with antibiotic sensitivity for each animal, as well as when planning the procurement of antibacterial drugs.

\section{Acknowledgements}

The research is executed at the expense of a grant of the RSF (project No. 18-16-00040)

\section{References}

1. Global Framework for Development \& Stewardship to Combat Antimicrobial Resistance. Draft Roadmap. WHO/EMP/ IAU/ 2017.08 (revised 19 October 2017) [Electronic resource]. URL: https:/www.who.int/antimicrobial-resistance/globalaction-plan/UpdatedRoadmap-Global-Framework-for-Development-Stewardship-to-combatAMR_2017_11_03.pdf?ua=1 (appeal date: 18.06.2019).

2. Order of the Government of the Russian Federation of September 25, 2017 No. 2045-r On the Strategy for preventing the spread of antimicrobial resistance in the Russian Federation for the period up to 2030. Strategy for preventing the spread of antimicrobial resistance in the Russian Federation for the period until 2030. Approved by the order of the Government of the Russian Federation of September 25, 2017 No. 2045-r [Electronic resource]. URL: https:/www.garant.ru/products/ipo/prime/ doc/71677266/\#review (appeal date: 15.06.2019)

3. Global action plan on antimicrobial resistance (Global action plan to combat antimicrobial resistance). SIXTY-EIGHTH WORLD HEALTH ASSEMBLY. WHA68.7. Agenda item 15.1 26 May 2015.). [Electronic resource] URL: https://apps.who. int/gb/ebwha/pdf files/WHA68/A68_R7-en.pdf?ua=1 (appeal date: 06.18.2019)

4. World Veterinary Association (WVA) Position on Responsible Use of Antimicrobials 2011. [Electronic resource]. URL: http://www.worldvet.org/uploads/docs/007_wva_position_on_antimicrobials_2011.pdf (appeal date: 11.06.2019).

5. Terrestrial Animal Health Code. Chapter 6.10. Responsible and prudent use of antimicrobial agents in veterinary medicine. 2019 C OIE [Electronic resource] URL: https://www.oie.int/fileadmin/Home/eng/Health_standards/tahc/current/chapitre_antibio_use.pdf (appeal date: 15.06.2019).

6. Kim D. H., Chung Y. S., Park Y. K., Yang S-J., Lim S. K., Park H. Y., Park K. T. Antimicrobial resistance and virulence profiles of Enterococcus spp. isolated from horses in Korea // Clinical Immunology, Microbiology and Infectious Disease. 2016. No. 48. Pp. 6-13. 


\section{Agrarian Bulletin of the Urals No. 09 (200), 2020}

7. Tong S. Y., Davis J. S., Eichenberger E., Holland T. L., Fowler V. G. Staphylococcus aureus infections: epidemiology, pathophysiology, clinical manifestations, and management // Clinical Microbiology Reviews. 2015. Vol. 28 (3). Pp. 603-661. 8. Shevchenko A. A., Chernykh O. Yu., Samuilenko A. Ya., Donnik I. M., Grin S. A., Shevchenko L. V., Shevkoplyas V. N., Koschaev A. G., Krivonogova A. S., Isaeva A. G., Krasochko P. A., Motuzko N. S. Diagnostics of infectious diseases of farm animals: bacterial diseases. Krasnodar: Kuban State Agrarian University named after I. T. Trubilina, 2018. 485 p.

9. Donnik I. M., Bykova O., Krivonogova A. S., Isaeva A. G., Loretts O. G., Baranova A., Musikhina H, Romanova A. Biological safety of cows' milk under the conditions of technogenic agricultural ecosphere when using biologically active substances // International Transaction Journal of Engineering, Management and Applied Sciences and Technologies. 2019. Vol. 10. No. 2. Pp. 203-209.

10. Ventola L. C. The antibiotic resistance crisis. Part I: causes and threats // Pharmacy and Therapeutics. 2015. No. 40. Pp. 1-3. 11. Lartseva L. V., Obukhova O. V., Barmin A. N. Environmental and biological danger of resistance of opportunistic microflora to antibiotics (review) // Russian Journal of Applied Ecology. 2015. No. 4 (4). Pp. 47-52.

12. Koba I. S., Lysenko A. A., Koshchaev A. G., Shantyz A. K., Donnik I. M., Dorozhkin V. I., Shabunin S. V. Prevention of mastitis in dairy cows on industrial farms // Journal of Pharmaceutical Sciences and Research. 2018. Vol. 10. No. 10. P. 2582

13. Khan M. F. Brief History of Staphylococcus aureus: A Focus to Antibiotic Resistance // EC Microbiology. 2017. No. 5. Pp. 36-39.

14. Wolter D. J., Lister P. D. Mechanisms of $\beta$-lactam resistance among Pseudomonas aeruginosa // Current Pharmaceutical Design. 2013. Vol. 19 (2). Pp. 209-222.

15. Rostami S., Sheikh A. F., Shoja S. Investigating of four main carbapenem-resistance mechanisms in high-level carbapenem resistant Pseudomonas aeruginosa isolated from burn patients // Journal of the Chinese Medical Association. 2018. Vol. 81 (2). Pp. 127-132.

16. Seixas R., Varanda D., Bexiga R., Ttavares L., Oliveira M. Biofilm-formation by Staphylococcus aureus and Staphylococcus epidermidis isolates from subclinical mastitis in conditions mimicking the udder environment // Polish Journal of Veterinary Sciences. 2015. Vol. 18. No. 4. Pp. 791-792.

17. Ilchenko E. L., Smirnova Yu. A. Study of the resistance of bacteria of the class staphylococcus to broad spectrum antibiotics // In the collection: New tasks of modern medicine: materials of the V International scientific conference. Kazan, 2018. Pp. 28-35.

18. Iweriebor B.C., Obi, L. C., Okoh, A. I. Macrolide, glycopeptide resistance and virulence genes in Enterococcus species isolates from dairy cattle // Journal of Medical Microbiology. 2016. Vol. 65. Pp. 641-648.

19. Von Wintersdorff, C. J. H., Penders J., van Niekerk J. M., Mills N. D., Majumder S., van Alphen L. B., Savelkoul P. H. M., Wolffs P. F. G. Dissemination of antimicrobial resistance in microbial ecosystems through horizontal gene transfer // Frontiers in Microbiology. 2016. Vol. 7. P. 173.

20. Chebotar I. V., Bocharova Yu. A., Mayansky N. A. Mechanisms of Pseudomonas aeruginosa resistance to antibiotics and their regulation // Clinical microbiology and antimicrobial chemotherapy. 2017. T. 19. No. 4. Pp. 308-331.

21. Clinical recommendations. Determination of the susceptibility of microorganisms to antimicrobials, 2015 [Electronic resource]. URL: http://www.antibiotic.ru/minzdrav/files/docs/clrec-dsma2018.pdf (appeal date: 21.06.2019).

\section{Authors'information:}

Anna S. Krivonogova ${ }^{1}$, doctor of biological sciences, associate professor, leading researcher, ORCID 0000-0003-1918-3030, AuthorID 683239; +7 982 651-29-34

Irina M. Donnik ${ }^{2}$, doctor of biological sciences, professor, academician of the Russian Academy of Sciences, head of the department of infectious and non-infectious pathology, ORCID 0000-0001-8349-3004, AuthorID 313786

Albina G. Isaeva ${ }^{2}$, doctor of biological sciences, professor of the department of infectious and non-infectious pathology, ORCID 0000-0001-8395-124, AuthorID 665717; +7 982 872-89-10

Ksenia V. Moiseeva ${ }^{2}$, assistant of the department of infectious and non-infectious pathology, ORCID 0000-0002-9858-1880, AuthorID 779572; +7 953 047-58-87

${ }^{1}$ Ural Federal Agrarian Research Center of the Ural Branch of the Russian Academy of Sciences, Ekaterinburg, Russia

${ }^{2}$ Ural State Agrarian University, Ekaterinburg, Russia 